\title{
Kidney function and other factors and their association with falls
}

\section{The screening for CKD among older people across Europe (SCOPE) study}

\author{
Sabine Britting ${ }^{1}$, Rada Artzi-Medvedik², Paolo Fabbietti ${ }^{3,4^{*}}$ (D), Lisanne Tap ${ }^{5}$, Francesco Mattace-Raso ${ }^{5}$, \\ Andrea Corsonello ${ }^{3}$, Fabrizia Lattanzio ${ }^{3}$, Johan Ärnlöv ${ }^{6,7,8}$, Axel C. Carlsson ${ }^{6,7}$, Regina Roller-Wirnsberger 9 , \\ Gerhard Wirnsberger $^{10}$, Tomasz Kostka ${ }^{11}$, Agnieszka Guligowska ${ }^{11}$, Francesc Formiga ${ }^{12}$, Rafael Moreno-Gonzalez ${ }^{12}$, \\ Pedro Gil ${ }^{13}$, Sara Lainez Martinez ${ }^{13}$, Robert Kob ${ }^{1}$, Itshak Melzer ${ }^{14 \dagger}$, Ellen Freiberger ${ }^{1 \dagger}$ and on behalf of the SCOPE \\ investigators
}

\begin{abstract}
Background: Reduced kidney function has become a major public health concern, especially among older people, as Chronic Kidney Disease (CKD) is associated with increased risk of end stage renal disease and mortality. Falls are a serious negative health outcome in older persons with one third of people aged 65 years experiencing a fall per year and increasing fall rates with increasing age. The impact of CKD on falls in older community-dwelling persons is not well investigated. Additionally, lower urinary tract symptoms (LUTS) may also increase the risk of falls. Therefore, our aim was to investigate the impact of CKD and LUTS on falls as well as on injurious falls.

Methods: The SCOPE study is an observational, multinational, multicenter, prospective cohort study involving communitydwelling older persons aged 75 years and more recruited from August 2016 to March 2018 in seven European countries. The main outcomes of the present study were any falls and any injurious falls during the 12 months before enrolment. The cross-sectional association of estimated glomerular filtration rate (eGFR) and LUTS with study outcomes was investigated by logistic regression analysis adjusted for baseline characteristics of enrolled subjects.
\end{abstract}

(Continued on next page)

\footnotetext{
* Correspondence: p.fabbietti@inrca.it

${ }^{+}$Itshak Melzer and Ellen Freiberger contributed equally to this work.

${ }^{3}$ Italian National Research Center on Aging (IRCCS INRCA), Fermo and Cosenza, Ancona, Italy

${ }^{4}$ Laboratory of Geriatric Pharmacoepidemiology and Biostatistics, IRCCS INRCA, Via S. Margherita 5, 60124 Ancona, Italy

Full list of author information is available at the end of the article
}

(C) The Author(s). 2020 Open Access This article is licensed under a Creative Commons Attribution 4.0 International License, which permits use, sharing, adaptation, distribution and reproduction in any medium or format, as long as you give appropriate credit to the original author(s) and the source, provide a link to the Creative Commons licence, and indicate if changes were made. The images or other third party material in this article are included in the article's Creative Commons licence, unless indicated otherwise in a credit line to the material. If material is not included in the article's Creative Commons licence and your intended use is not permitted by statutory regulation or exceeds the permitted use, you will need to obtain permission directly from the copyright holder. To view a copy of this licence, visit http://creativecommons.org/licenses/by/4.0/ The Creative Commons Public Domain Dedication waiver (http://creativecommons.org/publicdomain/zero/1.0/) applies to the data made available in this article, unless otherwise stated in a credit line to the data. 


\begin{abstract}
(Continued from previous page)
Results: Our series consisted of 2256 SCOPE participants (median age $=79.5$ years, 55.7\% female). Of them, 746 participants experienced a fall and 484 reported an injurious fall in the 12 months prior to baseline assessment. CKD was not significantly associated with falls $(O R=0.95,95 \% \mathrm{Cl}=0.79-1.14$ for eGFR $<60 ; O R=1.02,95 \% \mathrm{Cl}=0.81-1.28$ for $\mathrm{eGFR}<45 ; \mathrm{OR}=1.08$, $95 \% \mathrm{Cl}=0.74-1.57$ for eGFR<30) or injurious falls $(O R=0.91,95 \% \mathrm{Cl}=0.67-1.24$ for eGFR $<60 ; O R=0.93,95 \% \mathrm{Cl}=0.63-1.37$ for eGFR $<45 ; O R=1.19,95 \% \mathrm{Cl}=0.62-2.29$ for eGFR<30). LUTS were found significantly associated with both falls $(O R=1.56$, $95 \% \mathrm{Cl}=1.29-1.89)$ and injurious falls $(\mathrm{OR}=1.58,95 \% \mathrm{Cl}=1.14-2.19)$, and such associations were confirmed in all multivariable models.

Conclusions: Cross-sectional data suggest that CKD may not be associated with history of falls or injurious falls, whereas LUTS is significantly associated with the outcomes.
\end{abstract}

Trial registration: This study was registered on 25th February 2016 at clinicaltrials.gov (NCT02691546).

Keywords: Kidney function, Older people, Falls, Injurious falls, Fear of falling, Urinary incontinence

\section{Background}

Due to the demographic changes, new challenges arise for the public health care systems and in older persons themselves. Current clinical and public health approaches more and more try to sustain functional capacity and resilience, including functionality in an integrated care concept for all citizens and across lifespan. Major endpoints of adverse life trajectories are deteriorating chronic illnesses and geriatric syndromes. A fall or injurious fall threatens functionality and independence in older persons and are often the start of a downward spiral leading to nursing home admission or even death. Therefore, the International Consortium for Health Outcomes Measurement (ICHOM) included "falls" as a standard set to determine health outcome [1].

One third of persons older than 65 years experienced at least one fall per year and history of falling doubles the chances of additional falls [2]. Among communitydwelling people aged 80 or more the percentage of fallers increases up to $50 \%[3,4]$. Very often falls -based on different definitions- are accompanied by fractures [5], causing distress, pain and immobilization. In the US overall medical costs for fatal falls was estimated to be $\$ 754$ million [5]. As the older population still increases, the number of fall injuries and the cost to treat them will pose a tremendous burden to the health care systems.

Many falls do not cause injuries, but they have negative impact on quality of life [6] and independency in daily activities [7-9]. In the event of a fall, self-efficacy can decrease due to fall-related psychological concerns (FrPC). It has been reported earlier, that 21 and $85 \%$ of older adults suffer from FrPC, which may be seen as an independent risk factor for falls $[10,11]$. FrPC may lead to inactivity, decreased physical function, symptoms of depression and therefore reduced quality of life [12]. By nature, falls are defined as geriatric syndromes with a multi-causal nature. In this context, frailty, polypharmacy and chronic diseases, such as chronic kidney disease (CKD) have also been identified as factors promoting risk of falling [13].
Information on the impact of CKD on falls in older community-dwelling persons are rare. Among people aged 65 or more, Kistler et al. [14] demonstrated an increased odds ratio (1.81) for falls in CKD patients. This finding was confirmed by Paliwal et al. [15] in an US Population with an adjusted odds ratio of 1.27 (95\% CI 1.14-1.42). In the above studies blood and urine analysis were not performed, eGFR were not reported, and diagnosis of CKD was only based on clinical history.

Lower urinary tract symptoms (LUTS) also deserve to be considered when investigating the association between CKD and falls. In a recent small study by Lai et al. results demonstrated an elevated prevalence of urological diseases [16] in CKD older persons. Different pathways have been proposed for this relationship including medication as diuretics for hypertension, overactive bladder especially for women, or changes in the bladder urothelium [17]. Additionally, urinary incontinence has been demonstrated as a risk factor on falls $[18,19]$.

In this context, the SCOPE study provides a clinically relevant source of community-dwelling older adults to study the relationship between chronic diseases, such as CKD, functionality and the risk of falling in a population older than 75 years. The objectives of the present investigation were (1) to evaluate different intrinsic correlates of history of falling based upon self-report from participants at baseline; (2) to evaluate correlates of history of injurious falls at baseline retrospectively; (3) to explore the impact of CKD and LUTS on falls and injurious falls.

\section{Methods \\ Study design and participants}

The SCOPE study (European Grant Agreement no. 436849), is a multicenter 2-year prospective cohort study involving patients older than 75 years attending outpatient services in participating institutions in seven countries (Austria, Germany, Israel, Italy, the Netherlands, Poland and Spain). Methods of the SCOPE study have been extensively described elsewhere. Participants were requested 
to sign a written informed consent before entering the study. The study protocol was approved by ethics committees at all participating institutions, and complies with the Declaration of Helsinki and Good Clinical Practice Guidelines. Only baseline data are used in the present study. Exclusion criteria were: end-stage renal disease or dialysis at time of enrollment; history of solid organ or bone marrow transplantation; active malignancy within 24 months prior to screening or metastatic cancer; life expectancy less than 6 months (based on the judgment of the study physician after careful medical history collection and diagnoses emerging from examination of clinical documentation exhibited); severe cognitive impairment (Mini Mental State Examination < 10); any medical or other reason (e.g. known or suspected patients' inability to comply with the protocol procedure) in the judgement of the investigators, that the patient was unsuitable for the study; unwilling to provide consent and limited possibility to attend followup visits [20].

Overall, 2461 participants were initially enrolled in the study. Of them, 2256 participants were included in the analysis presented in the current publication and 205 participants had to be excluded from analysis due to incomplete baseline data collected between August 2016 to March 2018.

\section{Measures}

\section{Sociodemographic, anthropometry, laboratory analysis}

Data on history of falls and incident falls were collected retrospective via a face-to face interview questionnaire. For prevalence of falls and injurious falls, two questions were asked: 1) how many times have you fallen in the past 12 months, (answer "No" for 0 events, and the answer "yes" for 1 or more falls), and 2) what kind of injuries did you sustain from a fall (answer: (fractures, treated and untreated injury, and no injury). Further information of the location and circumstances of the fall were documented. An injurious fall was defined as a fall causing fractures, treated or untreated injuries. Fear of falling (FoF) was obtained with a single question with possible answers ranging from not at all concerned, somewhat concerned, fairly concerned and very concerned.

To assess the CKD stage, blood and urine analysis were performed by the locally certified laboratories adhering to the protocol. Serum creatinine was measured at local level by standard methods. Creatinine-based eGFR was calculated using the Berlin Initiative Study 1 (BIS1) equation, especially developed for persons 70 years and older [21] using serum creatinine levels. Three different severity threshold for CKD were used: $<60,<45$ and $<30 \mathrm{ml} / \mathrm{min} / 1.73 \mathrm{~m}^{2}$.

Lower urinary tract symptoms (LUTS) were assessed by asking the patient to rate on a 5 Likert point scale $(0-4)$ the presence of LUTS within the last 4 weeks [22]. Urinary incontinence was defined as at least one moderate or big problem in dripping or leaking urine, weak urine stream or incomplete emptying, waking up to urinate, need to urinate frequently during the day.

Participant's characteristics were assessed during the baseline interview and medical examination. Demographic variables included age, gender and self-reported living-status (living alone vs. living with others). Body mass index (BMI) was calculated by body weight and height and expressed as $\mathrm{kg} / \mathrm{m}^{2}$, measured according to standard operating procedures.

Comprehensive geriatric assessment (CGA) was performed including Mini Mental State Examination (MMSE)/ cognitive status [23], 15-items Geriatric Depression Scale (GDS)/mood [24], Basic (ADL) and Instrumental Activities of Daily Living (IADL)/self-reported disability [25, 26]. Physical function was assessed with the short physical performance battery (SPPB) [27] including gait speed, five chair-stands test (time to rise from a chair and return to the seated position 5 times without using arms) and balance test (ability to stand with the feet together in the side-byside, semi-tandem, and tandem positions).

Diseases were documented and analyzed using Cumulative Illness Rating Scale for Geriatrics (CIRS-G Total Score)/overall comorbidity [28]. The number of medications was also calculated and included in the analysis.

Health related quality of life was rated by Euro-Qol 5D [29] questionnaire, including total score and QoL visual analogue scale (EQ-VAS).

\section{Statistical analysis}

First, we compared participants grouped according to self-reported falls or injurious falls during the 12 months before enrolment. Chi-square test was used to compare categorical variables and Student T-test for continuous ones. In order to obtain an adjusted estimate of the association of eGFR or LUTS with study outcomes, we built logistic regression models adjusted for age, gender, SPPB score, GDS, IADL, Euro-Qol 5D. The analyses were also repeated by removing SPPB from multivariable models.

Statistical analysis was carried out using SPSS for Win V24.0 (SPSS Inc., Chicago, IL, USA). $p$-value of $<0.05$ was considered statistically significant.

\section{Results}

Overall, 2256 participants were included in the analysis (median age 79.5 years (5.9 interquartile range (IR), and $55.7 \%$ female) (Table 1). At least 1 fall was reported by 746 (33.1\%) participants. Of them, 484 (64.9\%) reported an injurious fall and 262 reported a non-injurious fall in the 12 months prior to baseline assessment.

\section{Fallers versus non-fallers}

Fallers were older and more frequently female compared to non-fallers. Living alone was more prevalent among fallers, who also exhibited higher number of medications, IADL score and Euro-Qol 5D Total score, as well as lower 
Table 1 Characteristics of the study population in total, in fallers and non-fallers reported retrospectively in the past year

\begin{tabular}{|c|c|c|c|c|}
\hline Variable & All $(N=2256)$ & No falls past 12 months $(N=1510)$ & At least 1 fall past 12 months $(N=746)$ & $p$ \\
\hline Age, years & $79.5(5.9)$ & $79.4(5.5)$ & $79.6(6.6)$ & 0.022 \\
\hline Gender (Female), n (\%) & $1256(55.7)$ & $795(52.6)$ & $461(61.8)$ & $<0.001$ \\
\hline Educational level, years & $11.0(7.0)$ & $11.0(7.0)$ & $11.0(6.0)$ & 0.973 \\
\hline Living alone, n (\%) & $551(24.4)$ & $341(22.6)$ & $210(28.2)$ & 0.004 \\
\hline MMSE & $29.0(3.0)$ & $29.0(3.0)$ & $29.0(3.0)$ & 0.212 \\
\hline GDS & $2.0(3.0)$ & $2.0(3.0)$ & $2.0(3.0)$ & $<0.001$ \\
\hline IADL & $2.0(9.0)$ & $2.0(8.0)$ & $3.0(11.0)$ & $<0.001$ \\
\hline Urinary incontinence (LUTS), n (\%) & $653(28.9)$ & $390(25.8)$ & $263(35.3)$ & $<0.001$ \\
\hline \multicolumn{5}{|c|}{ Euro-Qol 5D } \\
\hline Score & $7.0(4.0)$ & $7.0(3.0)$ & $8.0(4.0)$ & $<0.001$ \\
\hline "your health today" & $73.0(20.0)$ & $75.0(25.0)$ & $70.0(30.0)$ & $<0.001$ \\
\hline CIRS-G Total Score & $8.0(6.0)$ & $8.0(6.0)$ & $8.0(7.0)$ & $<0.001$ \\
\hline \multicolumn{5}{|l|}{ Comorbidities } \\
\hline Hypertension, n (\%) & $1733(76.8)$ & $1147(76.0)$ & $586(78.6)$ & 0.170 \\
\hline Stroke, n (\%) & $131(5.8)$ & $68(4.5)$ & $63(8.4)$ & $<0.001$ \\
\hline Hip fracture, n (\%) & $111(4.9)$ & $49(3.2)$ & $62(8.3)$ & $<0.001$ \\
\hline Osteoporosis, n (\%) & $688(30.5)$ & $423(28.0)$ & $265(35.5)$ & $<0.001$ \\
\hline Parkinson, n (\%) & $45(2.0)$ & $25(1.7)$ & $20(2.7)$ & 0.101 \\
\hline Diabetes, n (\%) & $568(25.2)$ & $369(24.4)$ & $199(26.7)$ & 0.249 \\
\hline Number of medications & $4.0(8.0)$ & $4.0(7.0)$ & $5.0(8.0)$ & $<0.001$ \\
\hline eGFR (BIS equation) & & & & 0.586 \\
\hline$\geq 60, \mathrm{n}(\%)$ & $832(36.9)$ & $551(36.5)$ & $281(37.7)$ & \\
\hline$<60, \mathrm{n}(\%)$ & $1424(63.1)$ & $959(63.5)$ & $465(62.3)$ & \\
\hline$<45, n(\%)$ & $561(24.9)$ & $369(24.2)$ & $192(25.7)$ & 0.862 \\
\hline$<30, n(\%)$ & $141(6.3)$ & $91(6.0)$ & $50(6.7)$ & 0.696 \\
\hline $\mathrm{BMl}, \mathrm{kg} / \mathrm{m}^{2}$ & $27.3(5.7)$ & $27.3(5.6)$ & $27.3(6.2)$ & 0.939 \\
\hline SPPB Total Score & $9.0(4.0)$ & $10.0(3.0)$ & $9.0(5.0)$ & $<0.001$ \\
\hline SPPB Balance & & & & $<0.001$ \\
\hline Severe limitation, n (\%) & $432(20.1)$ & $241(16.6)$ & $191(27.4)$ & \\
\hline Moderate limitation, n (\%) & $410(19.1)$ & $265(18.3)$ & $145(20.8)$ & \\
\hline No limitation, n (\%) & $1307(60.8)$ & $946(65.2)$ & $361(51.8)$ & \\
\hline SPPB Gait Speed & & & & $<0.001$ \\
\hline$\leq 8.70 \mathrm{~s}, \mathrm{n}(\%)$ & $2040(92.2)$ & $1393(93.7)$ & $647(89.0)$ & \\
\hline$>8.70 \mathrm{~s}, \mathrm{n}(\%)$ & $173(7.8)$ & $93(6.3)$ & $80(11.0)$ & \\
\hline
\end{tabular}

Note: values are expressed as percentage or median (IQR)

Abbreviations: $A D L$ activities of daily living, BIS Berlin Initiative Study, BMI body mass index, CIRS-G cumulative illness rating scale for geriatrics, eGFR estimated glomerular filtration rate, GDS geriatric depression scale, IADL instrumental activities of daily living, LUTS lower urinary tract symptoms, MMSE mini mental state examination, SSPB short physical performance battery

SPPB Total Score. LUTS were significantly more frequent in fallers than in non-fallers, while no significant difference was observed in regards to kidney function (Table 1).

\section{Injurious fallers versus non-injurious fallers}

Female gender and LUTS were more frequent among injurious vs non-injurious fallers. Injurious fallers also had higher Geriatric Depression Scale and IADL score, while SPPB Total score was lower in injurious vs non-injurious fallers. Even in this case, no significant difference was observed in regards to kidney function. Fair concern for falling was more frequent among injurious fallers (Table 2). 
Table 2 Characteristics of the study population and comparisons between retrospectively reported injurious falls versus noninjurious falls

\begin{tabular}{|c|c|c|c|c|}
\hline Variable & $\begin{array}{l}\text { All }(N= \\
746)\end{array}$ & $\begin{array}{l}\text { No injurious falls (Fractures/treated injury/ } \\
\text { untreated injury) } \\
(N=262)\end{array}$ & $\begin{array}{l}\text { At least } 1 \text { injurious fall (Fractures/treated injury/ } \\
\text { untreated injury) } \\
(N=484)\end{array}$ & $p$ \\
\hline Age, yrs. & $79.6(6.6)$ & $80.0(6.6)$ & $79.5(6.7)$ & 0.259 \\
\hline Gender (Female), n (\%) & $\begin{array}{l}461 \\
(61.8)\end{array}$ & $140(53.4$ & $321(66.3)$ & 0.001 \\
\hline Educational level, years & $11.0(6.0)$ & $11.0(6.0)$ & $11.0(7.0)$ & 0.569 \\
\hline Living alone, n (\%) & $210(28.2)$ & $71(27.1)$ & $139(28.7)$ & 0.639 \\
\hline MMSE score & $29.0(3.0)$ & $29.0(3.0)$ & $29.0(3.0)$ & 0.947 \\
\hline Geriatric Depression Score & $2.0(3.0)$ & $2.0(3.0)$ & $3.0(4.0)$ & 0.001 \\
\hline IADL & $\begin{array}{l}3.0 \\
(11.0)\end{array}$ & $2.0(10.0)$ & $4.0(11.0)$ & 0.046 \\
\hline $\begin{array}{l}\text { Urinary incontinence (LUTS), n } \\
(\%)\end{array}$ & $\begin{array}{l}263 \\
(35.3)\end{array}$ & $75(28.6)$ & $188(38.8)$ & 0.005 \\
\hline \multicolumn{5}{|l|}{ Euro-Qol 5D } \\
\hline Total Score & $8.0(4.0)$ & $8.0(4.0)$ & $8.0(5.0)$ & 0.002 \\
\hline "your health today" & $\begin{array}{l}70.0 \\
(30.0)\end{array}$ & $70.0(20.0)$ & $70.0(30.0)$ & 0.056 \\
\hline CIRS-G Total Score & $8.0(7.0)$ & $8.0(7.0)$ & $9.0(7.0)$ & 0.545 \\
\hline \multicolumn{5}{|l|}{ Comorbidities } \\
\hline Hypertension, n (\%) & $586(78.6)$ & $197(75.2)$ & $389(80.4)$ & 0.100 \\
\hline Stroke, n (\%) & $63(8.4)$ & $16(6.1)$ & $47(9.7)$ & 0.091 \\
\hline Hip fracture, n (\%) & $62(8.3)$ & $11(4.2)$ & $51(10.5)$ & 0.003 \\
\hline Osteoporosis, n (\%) & $\begin{array}{l}265 \\
(35.5)\end{array}$ & $74(28.2)$ & $191(39.5)$ & 0.002 \\
\hline Parkinson, n (\%) & $20(2.7)$ & $7(2.7)$ & $13(2.7)$ & 0.991 \\
\hline Diabetes, n (\%) & $199(26.7)$ & $76(29.0)$ & $123(25.4)$ & 0.289 \\
\hline Number of medications & $5.0(8.0)$ & $5.0(8.0)$ & $5.0(8.0)$ & 0.967 \\
\hline eGFR (BIS equation) & & & & 0.559 \\
\hline$\geq 60, \mathrm{n}(\%)$ & $281(37.7)$ & $95(36.3)$ & $186(38.4)$ & \\
\hline$<60, \mathrm{n}(\%)$ & $465(62.3)$ & $167(63.7)$ & $298(61.6)$ & \\
\hline$<45, n(\%)$ & $\begin{array}{l}192 \\
(25.7)\end{array}$ & $68(26.0)$ & $124(25.6)$ & 0.718 \\
\hline$<30, n(\%)$ & $50(6.7)$ & $15(5.7)$ & $35(7.2)$ & 0.598 \\
\hline $\mathrm{BMl}, \mathrm{kg} / \mathrm{m}^{2}$ & $27.3(6.2)$ & $27.1(6.4)$ & $27.3(6.0)$ & 0.877 \\
\hline SPPB Total Score & $9.0(5.0)$ & $9.0(5.0)$ & $8.5(6.0)$ & 0.032 \\
\hline SPPB Balance & & & & 0.184 \\
\hline Severe limitation, n (\%) & $191(27.4)$ & $56(23.1)$ & $135(29.7)$ & \\
\hline Moderate limitation, n (\%) & $145(20.8)$ & $53(21.9)$ & $92(20.2)$ & \\
\hline No limitation, n (\%) & $361(51.8)$ & $133(55.0)$ & $228(50.1)$ & \\
\hline SPPB Gait Speed & & & & 0.026 \\
\hline$\leq 8.70 \mathrm{~s}, \mathrm{n}(\%)$ & $\begin{array}{l}647 \\
(89.0)\end{array}$ & $235(92.5)$ & $412(87.1)$ & \\
\hline$>8.70 \mathrm{~s}, \mathrm{n}(\%)$ & $80(11.0)$ & $19(7.5)$ & $61(12.9)$ & \\
\hline
\end{tabular}


Table 2 Characteristics of the study population and comparisons between retrospectively reported injurious falls versus noninjurious falls (Continued)

\begin{tabular}{llll}
\hline Variable & All (N= & $\begin{array}{l}\text { No injurious falls (Fractures/treated injury/ } \\
\text { untreated injury) } \\
(N=262)\end{array}$ & $\begin{array}{l}\text { At least } 1 \text { injurious fall (Fractures/treated injury/ } \\
\text { untreated injury) } \\
(N=484)\end{array}$ \\
\hline $\begin{array}{l}\text { Fear of falling } \\
\begin{array}{l}\text { Single question: not at all } \\
\text { concerned }\end{array}\end{array}$ & $\begin{array}{l}337 \\
\mathbf{( 4 5 . 1 )}\end{array}$ & $\mathbf{1 4 1 ( 5 3 . 6 )}$ & $\mathbf{1 9 6 ( 4 0 . 5 )}$ \\
$\begin{array}{l}\text { Single question: fairly } \\
\text { concerned }\end{array}$ & $\begin{array}{l}\mathbf{1 1 8} \\
\mathbf{( 1 5 . 8 )}\end{array}$ & $\mathbf{2 6 ( 9 . 9 )}$ & $\mathbf{9 2 ( 1 9 . 0 )}$ \\
\hline
\end{tabular}

Note: values are expressed as percentage or median (IQR)

Abbreviations: $A D L$ activities of daily living, BIS Berlin Initiative Study, BMI body mass index, CIRS-G cumulative illness rating scale for geriatrics, eGFR estimated glomerular filtration rate, GDS geriatric depression scale, IADL instrumental activities of daily living, LUTS lower urinary tract symptoms, MMSE mini mental state examination, SSPB short physical performance battery

\section{Multivariable analysis}

In logistic regression analyses models no significant association was found between CKD and any falls (Table 3). The lack of significant association between CKD and falls was also confirmed when considering different CKD stages (e.g. $<30$ or $<45 \mathrm{ml} / \mathrm{min} / 1.73 \mathrm{~m}^{2}$ ) (Table 3). In model 2, female gender was significantly associated with the occurrence of any falls $(O R=1.45$, $95 \% C I=1.21-1.74)$. Such an association was also observed in all multivariable models (data not shown). Other significant correlates of the occurrence of any falls were SPPB $(O R=0.89,95 \% C I=0.86-0.92)$ in model 3, incontinence $(O R=1.37,95 \% C I=1.12-1.67)$ in model 4, and incontinence $(O R=1.33,95 \% C I=1.09-1.63)$, quality of life $(O R=1.03,95 \% C I=1.03-1.10)$, and IADL $(O R=1.02,95 \% C I=1.0-1.03)$. Similarly, CKD was not associated with injurious falls. Even in this case female gender was significantly associated with the outcome $(O R=1.70,95 \% C I=1.25-2.31)$ in model 2 , and this finding was confirmed in all multivariable models (data not shown). Other significant predictors of injurious falls were SPPB $(O R=0.95,95 \% C I=0.90-1.0)$ in model 3, incontinence $(O R=1.46,95 \%=1.04-2.05)$, GDS $(O R=$ $1.09,95 \% C I=1.02-1.17)$ and IADL $(O R=1.03,95 \% C I=$ $1.0-1.06)$ in model 4. Predictors in model 5 were almost identical to those in model 4 (data not shown). Notably, patients with more severe stages of CKD were older and more frequently male, had greater IADL dependency, CIRS-G score and number of medications, as well as lower SPPB total score and higher prevalence of hypertension, stroke and osteoporosis (Table 4).

Urinary incontinence (defined by the LUTS) was found associated with both any and injurious falls in all multivariable models (Table 5). Other predictors of any falls in this analysis were female gender $(O R=1.42,95 \% C I=$ 1.19-1.71) in model 2, SPPB $(O R=0.90,95 \% C I=0.87-$ $0.93)$ in model 3, SPPB $(O R=0.92,95 \% C I=0.88-0.95)$ in model 4, quality of life $(O R=1.06,95 \% C I=1.03$ $1.10)$ and IADL $(O R=1.02,95 \% C I=1.0-1.03)$ in model 5. Female gender was significantly associated with any falls in all multivariable models (data not shown). Female gender $(O R=1.66,95 \% C I=1.21-2.26)$ was significantly associated with injurious falls in model 2 , and such association was confirmed in all multivariable models (data not shown). Finally,

Table 3 Probability of at least 1 fall $(n=2256)$ and of at least 1 injurious fall $(n=746)$ in CKD groups, in the SCOPE cohort at baseline

\begin{tabular}{|c|c|c|}
\hline Predictors & $\begin{array}{l}\text { OR }(95 \% \mathrm{Cl}) \\
\text { At least } 1 \text { fall }\end{array}$ & $\begin{array}{l}\text { OR }(95 \% \mathrm{Cl}) \text { At least } 1 \text { injurious } \\
\text { fall }\end{array}$ \\
\hline Model 1. Compares CKD group (combining several stages in CKD) vs. non-CKD group & $0.95(0.79-1.14)$ & $0.91(0.67-1.24)$ \\
\hline Model 1.2 Compares CKD group (<45) vs. non-CKD group & $\begin{array}{l}1.02(0.81- \\
1.28)\end{array}$ & $0.93(0.63-1.37)$ \\
\hline Model 1.3 Compares CKD group $(<30)$ vs. non-CKD group & $\begin{array}{l}1.08(0.74- \\
1.57)\end{array}$ & $1.19(0.62-2.29)$ \\
\hline Model 2. Adjusted for age and gender & $0.91(0.75-1.10)$ & $0.97(0.70-1.34)$ \\
\hline Model 3. Adjusted for age, gender and SPPB total score & $0.84(0.69-1.01)$ & $0.93(0.67-1.28)$ \\
\hline $\begin{array}{l}\text { Model 4. Adjusted for age, gender, SPPB total score, GDS, incontinence, IADL score and Euro-Qol } \\
\text { 5D Score }\end{array}$ & $0.84(0.69-1.02)$ & $0.92(0.66-1.28)$ \\
\hline Model 5. Adjusted for age, gender, GDS, incontinence, IADL score and Euro-Qol 5D Score & $0.87(0.72-1.05)$ & ${ }^{\mathrm{a}} 0.94(0.68-1.32)$ \\
\hline
\end{tabular}

Note: ${ }^{2} 2$ "fear of falling" variables were added (not at all concerned vs other; fairly concerned vs other)

Abbreviations: $C l$ confidence interval, $C K D$ chronic kidney disease, GDS geriatric depression score, IADL instrumental activities of daily living, $O R$ odds ratio, SPPB short physical performance battery 
Table 4 Significant correlates of eGFR categories in the patients studied

\begin{tabular}{|c|c|c|c|c|c|}
\hline Variable & $\begin{array}{l}\text { eGFR }>60 \\
N=\ldots\end{array}$ & $\begin{array}{l}\text { eGFR 45-60 } \\
N=\ldots\end{array}$ & $\begin{array}{l}\text { eGFR 30-45 } \\
N=\ldots\end{array}$ & $\begin{array}{l}\text { eGFR }<30 \\
N=\ldots\end{array}$ & $p$ \\
\hline Age, years & $78.6(4.5)$ & $79.6(5.7)$ & $80.9(6.8)$ & $81.0(7.2)$ & $<0.001$ \\
\hline Gender (Female), n (\%) & $508(61.1)$ & $490(56.8)$ & $201(47.9)$ & $57(40.4)$ & $<0.001$ \\
\hline IADL & $0.0(6.0)$ & $2.0(8.0)$ & $6.0(12.0)$ & $12.0(14.0)$ & $<0.001$ \\
\hline CIRS-G Total Score & $7.0(6.0)$ & $7.0(5.0)$ & $10.0(7.0)$ & $11.5(7.2)$ & $<0.001$ \\
\hline \multicolumn{6}{|l|}{ Comorbidities } \\
\hline Hypertension, n (\%) & $552(66.3)$ & $670(77.6)$ & $375(89.3)$ & $136(96.5)$ & $<0.001$ \\
\hline Stroke, n (\%) & $36(4.3)$ & $49(5.7)$ & $33(7.9)$ & $13(9.2)$ & 0.022 \\
\hline Osteoporosis, n (\%) & $247(29.7)$ & $255(29.5)$ & $122(29.0)$ & $64(45.4)$ & 0.001 \\
\hline Diabetes, n (\%) & $162(19.5)$ & $195(22.6)$ & $160(38.1)$ & $51(36.2)$ & $<0.001$ \\
\hline Number of medications & $2.0(6.0)$ & $4.0(7.0)$ & $6.0(7.0)$ & $7.0(5.0)$ & $<0.001$ \\
\hline SPPB Total Score & $10.0(3.0)$ & $10.0(4.0)$ & $8.0(6.0)$ & $7.0(5.0)$ & $<0.001$ \\
\hline
\end{tabular}

Note: values are expressed as percentage or median (IQR)

Abbreviations: CIRS-G cumulative illness rating scale for geriatrics, eGFR estimated glomerular filtration rate, IADL instrumental activities of daily living, SPPB short physical performance battery

GDS $(O R=1.09,95 \% C I=1.02-1.17)$ and IADL $(O R=1.03$, $95 \% C I=1.0-1.06)$ also qualified as significant correlates of injurious falls in model 4 , and almost identical findings were observed in model 5 (data not shown).

\section{Discussion}

In this paper, being old and female are predictors of falls as well as injurious falls. This results are controversial since Clemson et al. [30], found that men were at higher risk of falls while Todd and Skelton [18] found that female were at higher risk of falls. In our cohort about $38 \%$ of men and $61.8 \%$ of women experienced a fall. In line with former studies $[18,19]$ are our findings that women are at higher risk of falls. We also found that falls and injurious falls were related to lower physical function and self-reported disability (obtained with the
SPPB Total Score, decreased IADL Score and depression). This was in agreement with Clemson and colleagues [30], who found that injurious falls in the old age, were associated with lower physical function and depression. In addition, Gunter et al. [31] suggested that physical performance tests differed between people with and without a history of falls. Similarly, to Clemson et al. [30], we found a decreased quality of life and living alone as variables that are associated with falls. While Clemson et al. [30] found that FoF did not predict injurious falls, we found that the FoF in fallers and injured fallers was significantly higher than in non-fallers and non-injurious fallers respectively.

In our regression analysis, CKD defined by eGFR did not predict falls or injurious falls. Past studies have shown controversial results. While Kistler et al. [14] and Paliwal

Table 5 Probability of at least 1 fall $(n=2256)$ and of at least 1 injurious fall $(n=746)$ in continence/incontinence groups, in the SCOPE cohort at baseline

\begin{tabular}{|c|c|c|}
\hline Predictors & $\begin{array}{l}\text { OR }(95 \% \mathrm{Cl}) \\
\text { At least } 1 \text { fall }\end{array}$ & $\begin{array}{l}\text { OR }(95 \% \mathrm{Cl}) \text { At least } 1 \\
\text { injurious fall }\end{array}$ \\
\hline $\begin{array}{l}\text { Model 1. Compares incontinence group (at least } 1 \text { moderate or big problem in dripping or leaking urine, } \\
\text { weak urine stream or incomplete emptying, waking up to urinate, need to urinate frequently during the } \\
\text { day) vs. non incontinence group (other) }\end{array}$ & $\begin{array}{l}1.56 \text { (1.29- } \\
1.89)\end{array}$ & $1.58(1.14-2.19)$ \\
\hline Model 2. Adjusted for age and gender & $\begin{array}{l}1.51(1.25- \\
1.83)\end{array}$ & $1.55(1.12-2.15)$ \\
\hline Model 3. Adjusted for age, gender and SPPB total score & $\begin{array}{l}1.46(1.20- \\
1.77)\end{array}$ & $1.52(1.10-2.12)$ \\
\hline Model 4. Adjusted for age, gender, SPPB total score, GDS, CKD, IADL score and Euro-Qol 5D Score & $\begin{array}{l}1.37(1.11- \\
1.67)\end{array}$ & $1.46(1.04-2.05)$ \\
\hline Model 5. Adjusted for age, gender, GDS, CKD, IADL score and Euro-Qol 5D Score & $\begin{array}{l}1.33(1.09- \\
1.63)\end{array}$ & ${ }^{\mathrm{a}} 1.47(1.04-2.07)$ \\
\hline
\end{tabular}

Note: ${ }^{\text {a } 2 ~ " f e a r ~ o f ~ f a l l i n g " ~ v a r i a b l e s ~ w e r e ~ a d d e d ~(n o t ~ a t ~ a l l ~ c o n c e r n e d ~ v s ~ o t h e r ; ~ f a i r l y ~ c o n c e r n e d ~ v s ~ o t h e r) ~}$ Abbreviations: $C l$ confidence interval, $C K D$ chronic kidney disease, GDS geriatric depression score, IADL instrumental activities of daily living, $O R$ odds ratio, SPPB short physical performance battery 
et al. [15] demonstrated an increased odds ratio for falls in relation to CKD defined on the basis of clinical history and without reporting eGFR, a recent meta-analysis by Goto et al. [32] could not find a clear relationship between eGFR values and accidental fall risk. Nevertheless, a trend for worsening eGFR increasing accidental falls risk was observed, as well as a significant association between low eGFR and fractures resulting from accidental falls [32]. Additionally, CKD may not represent a risk factor on itself, but rather associated variables, e.g. reduced functional status, may mediate its detrimental effects [32]. The finding that patients with more severe stages of CKD are associated with coexisting conditions (e.g. IADL dependency and low SPPB score) which were found associated with falls in our analysis is in keeping with such hypothesis. Additionally, it is also important considering that present study shows cross-sectional data, and prospective analysis also including worsening CKD status as a risk factor may lead to different results.

In the SCOPE participants, about 33\% of participants had experienced at least one fall and of these $35 \%$ had experienced an injurious fall, which generally resembles former studies. Falls or injurious falls are related to a decreased quality of life, and lower physical function. Therefore, it is of importance to nephrologists to keep in mind that their older CKD patients might have a higher fall risk especially their older female patients. International recommendations require all health care professionals to ask their patients 65 years and older annually about falls history or unsteadiness in balance [33]. The STEADI toolkit from the Center for Chronic Disease [34] supports and helps to identify older persons with higher risk of falling. The early identification of older persons at risk of falls is important and helps to reduce medical costs [35], and thus relieve the public health care systems. Therefore, nephrologist normally dealing with kidney function should be aware of the fall risk in their older patients and ways of treatments.

We found that prevalence of LUTS is significantly higher in fallers vs non-fallers, and such association was confirmed in all multivariable models. Current evidence about the association of LUTS and injurious falls is controversial. In a 2-years follow-up study Schluter et al. [36] showed that for men and women urinary incontinence is not a risk factor for hip fractures, but for falls. However, the association of urinary incontinence and falls was described in several studies $[2,37,38]$, and urinary incontinent patients have a higher rate of falls and recurrent falls [36, 39-41]. Several studies addressing urinary incontinence included only women [38, 39, 42], whereas urinary incontinence occurs in both men and women [43]. Additionally, a systematic review by Nogouchi et al. [44] showed that urinary incontinence and LUTS was significantly associated with falls in community-dwelling older men. In our study, female gender was significantly associated with falls and injurious falls in LUTS analysis. Nevertheless, adjusting for gender did not change the strength of the observed association. We need to recognize the limited comparability of our findings to those obtained in former studies due to differences in methods for the assessment of LUTS and/or urinary incontinence. Additionally, urinary incontinence was found associated with risk of falling and fracture in community dwelling older women [39], while we included all injurious falls the patients experienced (treated and untreated injuries) not only fractures in our study. Anyway, findings from the present study suggest that increased likelihood of falls and injurious falls in relation to LUTS apply to both men and women.

\section{Limitations and strengths}

In the present study, some limitations warrant consideration. Falls data were collected by self-reported questionnaire retrospectively, with a possible over- or underestimation of falls. This is a cross-sectional study, and prospective analysis may yield different results. Falls follow-up studies are needed to determine whether eGFR values or worsening kidney function over time may increase the risk of falls. Third, the data came from a sample that was drawn from a relatively healthy community-based population; therefore, these results cannot be generalized to frail or institutionalized older persons.

An important strength of this study is the inclusion of participants of a community-dwelling population in reallife setting. Furthermore, we considered a wide set of potential confounders. In addition, the strict differentiation between falls and injurious falls enhance current understanding and will provide further evidence over the longitudinal follow-up.

\section{Conclusions}

LUTS may be cross-sectionally associated with increased likelihood of falls and injurious falls in communitydwelling people aged 75 or more, whereas CKD may not be associated with these outcomes. While the association between CKD and falls deserves to be prospectively investigated, treating LUTS and physical limitation might be an appropriate way to reduce risk of falling among older people, whatever is their kidney function.

\section{Abbreviations \\ CIRS-G Total Score: Cumulative IIIness Rating Scale for Geriatrics; \\ Cl: Confidence Interval; CKD: Chronic Kidney Disease; eGFR: Estimated Glomerular filtration rate; FoF: Fear of Falling; FrPC: Fall-related psychological concern; GDS: Geriatric Depression Scale; IADL: Instrumental Activity of Daily Living; MMSE: Mini-Mental State Examination; OR: Odds ratio; SPPB: Short Physical Performance Battery}

Acknowledgements

We thank the BioGer IRCCS INRCA Biobank for the collection of the SCOPE samples.

SCOPE study investigators. 
Coordinating center, Fabrizia Lattanzio, Italian National Research Center on Aging (INRCA), Ancona, Italy - Principal Investigator. Andrea Corsonello, Silvia Bustacchini, Silvia Bolognini, Paola D'Ascoli, Raffaella Moresi, Giuseppina Di Stefano, Cinzia Giammarchi, Anna Rita Bonfigli, Roberta Galeazzi, Federica Lenci, Stefano Della Bella, Enrico Bordoni, Mauro Provinciali, Robertina Giacconi, Cinzia Giuli, Demetrio Postacchini, Sabrina Garasto, Annalisa Cozza, Francesco Guarasci, Sonia D'Alia - Italian National Research Center on Aging (INRCA), Ancona, Fermo and Cosenza, Italy - Coordinating staff. Romano Firmani, Moreno Nacciariti, Mirko Di Rosa, Paolo Fabbietti - Technical and statistical support.

Participating centers

- Department of Internal Medicine, Medical University of Graz, Austria: Gerhard Hubert Wirnsberger, Regina Elisabeth Roller-Wirnsberger, Carolin Herzog, Sonja Lindner

- Section of Geriatric Medicine, Department of Internal Medicine, Erasmus MC, University Medical Center Rotterdam, The Netherlands: Francesco Mattace-Raso, Lisanne Tap, Gijsbertus Ziere, Jeannette Goudzwaard. - Department of Geriatrics, Healthy Ageing Research Centre, Medical University of Lodz, Poland: Tomasz Kostka, Agnieszka Guligowska, Łukasz Kroc, Bartłomiej K Sołtysik, Małgorzata Pigłowska, Agnieszka Wójcik, Zuzanna Chrząstek, Natalia Sosowska, Anna Telążka, Joanna Kostka, Elizaveta Fife, Katarzyna Smyj, Kinga Zel.

- The Recanati School for Community Health Professions at the faculty of Health Sciences at Ben-Gurion University of the Negev, Israel: Rada ArtziMedvedik, Yehudit Melzer, Mark Clarfield, Itshak Melzer; and Maccabi Healthcare services southern region, Israel: Rada Artzi-Medvedik, Ilan Yehoshua, Yehudit Melzer.

- Geriatric Unit, Internal Medicine Department and Nephrology Department Hospital Universitari de Bellvitge, Institut d'Investigació Biomèdica de Bellvitge - IDIBELL, L'Hospitalet de Llobregat, Barcelona, Spain: Francesc Formiga, Rafael Moreno-González, Xavier Corbella, Yurema Martínez, Carolina Polo, Josep Maria Cruzado.

- Department of Geriatric Medicine, Hospital Clínico San Carlos, Madrid: Pedro Gil Gregorio, Sara Laínez Martínez, Mónica González Alonso, Jose A. Herrero Calvo, Fernando Tornero Molina, Lara Guardado Fuentes, Pamela Carrillo García, María Mombiedro Pérez.

- Department of General Internal Medicine and Geriatrics, Krankenhaus Barmherzige Brüder Regensburg and Institute for Biomedicine of Aging, Friedrich-Alexander-Universität Erlangen-Nürnberg, Germany: Alexandra Renz, Susanne Muck, Stephan Theobaldy, Andreas Bekmann, Revekka Kaltsa, Sabine Britting, Robert Kob, Christian Weingart, Ellen Freiberger, Cornel Sieber.

- Department of Medical Sciences, Uppsala University, Sweden: Johan Ärnlöv, Axel Carlsson, Tobias Feldreich.

We thank the BioGer IRCCS INRCA Biobank for the collection of the SCOPE samples.

Scientific advisory board (SAB).

Roberto Bernabei, Catholic University of Sacred Heart, Rome, Italy. Christophe Bula, University of Lausanne, Switzerland.

Hermann Haller, Hannover Medical School, Hannover, Germany. Carmine Zoccali, CNR-IBIM Clinical Epidemiology and Pathophysiology of Renal Diseases and Hypertension, Reggio Calabria, Italy.

\section{Data and Ethics Management Board (DEMB).}

Dr. Kitty Jager, University of Amsterdam, The Netherlands.

Dr. Wim Van Biesen, University Hospital of Ghent, Belgium.

Paul E. Stevens, East Kent Hospitals University NHS Foundation Trust, Canterbury, United Kingdom.

\section{About this supplement}

This article has been published as part of BMC Geriatrics Volume 20 Supplement 1 2020: The Screening for Chronic Kidney Disease among Older People across Europe (SCOPE) project: findings from cross-sectional analysis. The full contents of the supplement are available at https://bmcgeriatr.biomedcentral.com/articles/supplements/volume-20-supplement-1.

\section{Authors' contributions}

SB: data collection, manuscript drafting and revision. EF \& IM: participated in study protocol design, data collection, manuscript drafting and revision. RAM: data collection, manuscript drafting and revision. PF: data management and statistical analyses, manuscript drafting and revision. FL, AC: conceived the study, coordinated study protocol and data collection, participated in manuscript drafting and revision. FM, LT, JÄ, ACC, RRW, GW, TK, AG, FF, RMG,
PG, SLM: participated in study protocol design, Data collection, and manuscript drafting and revision. All authors read and approved the final manuscript.

\section{Funding}

SCOPE study and publication costs are funded by the European Union Horizon 2020 program, under the Grant Agreement $n^{\circ}$ 634869. Funding body had no role in the design of the study and collection, analysis, and interpretation of data, writing the manuscript and in the decision to publish the results.

\section{Availability of data and materials}

Data will be available for SCOPE researchers through the project website (www.scopeproject.eu).

\section{Ethics approval and consent to participate}

The study protocol was approved by ethics committees at all participating institutions, and complies with the Declaration of Helsinki and Good Clinical Practice Guidelines. All patients signed a written informed consent to be enrolled. Only baseline data are used in the present study. Ethics approvals have been obtained by Ethics Committees in participating institutions as follows:

Italian National Research Center on Aging (INRCA), Italy, \#2015 0522 IN, January 27, 2016.University of Lodz, Poland, \#RNN/314/15/KE, November 17, 2015. Medizinische Universität Graz, Austria, \#28-314 ex 15/16, August 5, 2016. Erasmus Medical Center Rotterdam, The Netherland, \#MEC-2016-036 \#NL56039.078.15, v.4, March 7, 2016.

Hospital Clínico San Carlos, Madrid, Spain, \# 15/532-E_BC, September 16, 2016.

Bellvitge University Hospital Barcellona, Spain, \#PR204/15, January 29, 2016. Friedrich-Alexander University Erlangen-Nürnberg, Germany, \#340_15B, January 21, 2016

Helsinki committee in Maccabi Healthcare services, Bait Ba-lev, Bat Yam, Israel, \#45/2016, July 24, 2016.

\section{Consent for publication}

Not applicable.

\section{Competing interests}

The authors declare no competing of interest. The funder had no role in the design of the study; in the collection, analyses, or interpretation of data; in the writing of the manuscript, or in the decision to publish the results. Patients were requested to sign a written informed consent before entering the study.

\section{Author details}

${ }^{1}$ Department of Internal Medicine-Geriatrics, Institute for Biomedicine of Aging (IBA), Friedrich-Alexander-Universität Erlangen-Nürnberg, Erlangen, Germany. ${ }^{2}$ Department of Nursing, Recanati School for Community Health Professions at the faculty of Health Sciences, Ben-Gurion University of the Negev, Beersheba, Israel. ${ }^{3}$ Italian National Research Center on Aging (IRCCS INRCA), Fermo and Cosenza, Ancona, Italy. ${ }^{4}$ Laboratory of Geriatric Pharmacoepidemiology and Biostatistics, IRCCS INRCA, Via S. Margherita 5, 60124 Ancona, Italy. ${ }^{5}$ Department of Internal Medicine, Section of Geriatric Medicine, Erasmus University Medical Center Rotterdam, Rotterdam, The Netherlands. ${ }^{6}$ Department of Medical Sciences, Uppsala University, Uppsala, Sweden. ${ }^{7}$ Division of Family Medicine, Department of Neurobiology, Care Sciences and Society, Karolinska Institutet, Stockholm, Sweden. ${ }^{8}$ School of Health and Social Studies, Dalarna University, Falun, Sweden. ${ }^{9}$ Department of Internal Medicine-Geriatrics, Medical University of Graz, Graz, Austria. ${ }^{10}$ Division of Nephrology, Department of Internal Medicine, Medical University of Graz, Graz, Austria. ${ }^{11}$ Department of Geriatrics, Healthy Ageing Research Centre, Medical University of Lodz, Lodz, Poland. ${ }^{12}$ Geriatric Unit, Internal Medicine Department, Bellvitge University Hospital - IDIBELL L'Hospitalet de Llobregat, Barcelona, Spain. ${ }^{13}$ Geriatric Department, Hospital Clínico San Carlos, Martín Lagos S/N, 28040 Madrid, Spain. ${ }^{14}$ Department of Physical Therapy, Recanati School for Community Health Professions at the faculty of Health Sciences, Ben-Gurion University of the Negev, Beersheba, Israel. 
Received: 5 August 2020 Accepted: 11 August 2020

Published: 2 October 2020

\section{References}

1. Akpan A, Roberts C, Bandeen-Roche K, Batty B, Bausewein C, Bell D, Bramley D, Bynum J, Cameron ID, Chen LK, et al. Standard set of health outcome measures for older persons. BMC Geriatr. 2018;18(1):36.

2. O'Loughlin $J$, Robitaille Y, Boivin JF, Suissa S. Incidence of and risk factors for falls and injurious falls among the community-dwelling elderly. Am J Epidemiol. 1993;137(3):342-54.

3. Campbell AJ, Borrie MJ, Spears GF, Jackson SL, Brown JS, Fitzgerald JL. Circumstances and consequences of falls experienced by a community population 70 years and over during a prospective study. Age Ageing. 1990; 19(2):136-41.

4. Rubenstein LZ. Falls in older people: epidemiology, risk factors and strategies for prevention. Age Ageing. 2006;35(Suppl 2):ii37-41.

5. Florence CS, Bergen G, Atherly A, Burns E, Stevens J, Drake C. Medical costs of fatal and nonfatal falls in older adults. J Am Geriatr Soc. 2018;66(4):693-8.

6. Schoene D, Heller C, Aung YN, Sieber CC, Kemmler W, Freiberger E. A systematic review on the influence of fear of falling on quality of life in older people: is there a role for falls? Clin Interv Aging. 2019;14:701-19.

7. Lusardi MM, Fritz S, Middleton A, Allison L, Wingood M, Phillips E, Criss M, Verma S, Osborne J, Chui KK. Determining risk of falls in community dwelling older adults: a systematic review and meta-analysis using posttest probability. J Geriatr Phys Ther. 2017;40(1):1-36.

8. Denkinger MD, Lukas A, Nikolaus T, Hauer K. Factors associated with fear of falling and associated activity restriction in community-dwelling older adults: a systematic review. Am J Geriatr Psychiatry. 2015;23(1):72-86.

9. Tinetti ME, Williams CS. Falls, injuries due to falls, and the risk of admission to a nursing home. N Engl J Med. 1997;337(18):1279-84.

10. Scheffer AC, Schuurmans MJ, van Dijk N, van der Hooft T, de Rooij SE. Fear of falling: measurement strategy, prevalence, risk factors and consequences among older persons. Age Ageing. 2008;37(1):19-24.

11. Delbaere K, Crombez G, Vanderstraeten G, Willems T, Cambier D. Fearrelated avoidance of activities, falls and physical frailty. A prospective community-based cohort study. Age Ageing. 2004;33(4):368-73.

12. Payette MC, Belanger C, Leveille V, Grenier S. Fall-related psychological concerns and anxiety among community-dwelling older adults: systematic review and meta-analysis. PLoS One. 2016;11(4):e0152848.

13. Morin L, Calderon Larranaga A, Welmer AK, Rizzuto D, Wastesson JW, Johnell K. Polypharmacy and injurious falls in older adults: a nationwide nested case-control study. Clin Epidemiol. 2019;11:483-93.

14. Kistler BM, Khubchandani J, Jakubowicz G, Wilund K, Sosnoff J. Falls and fallrelated injuries among US adults aged 65 or older with chronic kidney disease. Prev Chronic Dis. 2018;15:E82.

15. Paliwal Y, Slattum PW, Ratliff SM. Chronic health conditions as a risk factor for falls among the community-dwelling US older adults: a zero-inflated regression modeling approach. Biomed Res Int. 2017:2017:5146378.

16. Lai S, Pastore S, Piloni L, Mangiulli M, Esposito Y, Pierella F, Galani A, Pintus G, Mastroluca D, Shahabadi H, et al. Chronic kidney disease and urological disorders: systematic use of uroflowmetry in nephropathic patients. Clin Kidney J. 2018:12(3):414-9.

17. Chen SF, Lee $\mathrm{CL}$, Kuo HC. Changes in sensory proteins in the bladder urothelium of patients with chronic kidney disease and end-stage renal disease. Low Urin Tract Symptoms. 2019;11(2):0202-0208.

18. Todd C, Skelton D. What are the main risk factors for falls among older people and what are the most effecxtive interventions to prevent these falls? Copenhagen: WHO REgional Office for Europe; 2004.

19. Ambrose AF, Paul G, Hausdorff JM. Risk factors for falls among older adults: a review of the literature. Maturitas. 2013;75(1):51-61.

20. Corsonello A, Tap L, Roller-Wirnsberger R, Wirnsberger G, Zoccali C, Kostka T, Guligowska A, Mattace-Raso F, Gil P, Fuentes LG, et al. Design and methodology of the screening for CKD among older patients across Europe (SCOPE) study: a multicenter cohort observational study. BMC Nephrol. 2018;19(1):260

21. Schaeffner ES, Ebert N, Delanaye P, Frei U, Gaedeke J, Jakob O, Kuhlmann MK Schuchardt M, Tolle M, Ziebig R, et al. Two novel equations to estimate kidney function in persons aged 70 years or older. Ann Intern Med. 2012;157(7):471-81.

22. Rosenberg MT, Staskin DR, Kaplan SA, MacDiarmid SA, Newman DK, Ohl DA. A practical guide to the evaluation and treatment of male lower urinary tract symptoms in the primary care setting. Int J Clin Pract. 2007;61(9):1535-46.
23. Folstein MF, Folstein SE, McHugh PR. "Mini-mental state". A practical method for grading the cognitive state of patients for the clinician. J Psychiatr Res. 1975;12(3):189-98.

24. Lesher EL, Berryhill JS. Validation of the geriatric depression scale--short form among inpatients. J Clin Psychol. 1994;50(2):256-60.

25. Lawton MP, Brody EM. Assessment of older people: self-maintaining and instrumental activities of daily living. Gerontologist. 1969;9(3):179-86.

26. Katz $\mathrm{S}$, Ford AB, Moskowitz RW, Jackson BA, Jaffe MW. Studies of illness in the aged. The index of Adl: a standardized measure of biological and psychosocial function. JAMA. 1963;185:914-9.

27. Guralnik JM, Simonsick EM, Ferrucci L, Glynn RJ, Berkman LF, Blazer DG Scherr PA, Wallace RB. A short physical performance battery assessing lower extremity function: association with self-reported disability and prediction of mortality and nursing home admission. J Gerontol. 1994;49(2):M85-94.

28. Conwell Y, Forbes NT, Cox C, Caine ED. Validation of a measure of physica illness burden at autopsy: the cumulative illness rating scale. J Am Geriatr Soc. 1993:41(1):38-41.

29. Choi CW, Kim KH, Chang SS, Choi NJ. Soluble non-ammonia nitrogen in Ruminal and Omasal Digesta of Korean native steers supplemented with soluble proteins. Asian Australas J Anim Sci. 2012;25(9):1269-75.

30. Clemson L, Kendig H, Mackenzie L, Browning C. Predictors of injurious falls and fear of falling differ: an 11-year longitudinal study of incident events in older people. J Aging Health. 2015;27(2):239-56.

31. Gunter KB, White KN, Hayes WC, Snow CM. Functional mobility discriminates nonfallers from one-time and frequent fallers. J Gerontol A Biol Sci Med Sci. 2000:55(11):M672-6.

32. Goto NA, Weststrate ACG, Oosterlaan FM, Verhaar MC, Willems HC, Emmelot-Vonk MH, Hamaker ME. The association between chronic kidney disease, falls, and fractures: a systematic review and meta-analysis. Osteoporos Int. 2019.

33. Eckstrom E, Parker EM, Lambert GH, Winkler G, Dowler D, Casey CM. Implementing STEADI in Academic Primary Care to Address Older Adult Fall Risk. Innov Aging. 2017;1 (2):igx028.

34. Stevens JA, Phelan EA. Development of STEADI: a fall prevention resource for health care providers. Health Promot Pract. 2013;14(5):706-14.

35. Stevens JA, Lee R. The potential to reduce falls and avert costs by clinically managing fall risk. Am J Prev Med. 2018:55(3):290-7.

36. Schluter PJ, Arnold EP, Jamieson HA. Falls and hip fractures associated with urinary incontinence among older men and women with complex needs: a national population study. Neurourol Urodyn. 2018;37(4):1336-43.

37. Tinetti ME, Speechley M, Ginter SF. Risk factors for falls among elderly persons living in the community. N Engl J Med. 1988;319(26):1701-7.

38. Teo JS, Briffa NK, Devine A, Dhaliwal SS, Prince RL. Do sleep problems or urinary incontinence predict falls in elderly women? Aust J Physiother. 2006;52(1):19-24.

39. Brown JS, Vittinghoff E, Wyman JF, Stone KL, Nevitt MC, Ensrud KE, Grady D. Urinary incontinence: does it increase risk for falls and fractures? Study of osteoporotic fractures research group. J Am Geriatr Soc. 2000;48(7):721-5.

40. Hasegawa J, Kuzuya M, Iguchi A. Urinary incontinence and behavioral symptoms are independent risk factors for recurrent and injurious falls, respectively, among residents in long-term care facilities. Arch Gerontol Geriatr. 2010:50(1):77-81.

41. Kim HJ, Kim JW, Jang SN, Kim KD, Yoo Jl, Ha YC. Urinary incontinences are related with fall and fragility fractures in elderly population: Nationwide cohort study. J Bone Metab. 2018;25(4):267-74.

42. Takazawa K, Arisawa K. Relationship between the type of urinary incontinence and falls among frail elderly women in Japan. J Med Investig. 2005:52(3-4):165-71

43. Linde JM, Nijman RJM, Trzpis M, Broens PMA. Urinary incontinence in the Netherlands: prevalence and associated risk factors in adults. Neurourol Urodyn. 2017:36(6):1519-28.

44. Noguchi N, Chan L, Cumming RG, Blyth FM, Naganathan V. A systematic review of the association between lower urinary tract symptoms and falls, injuries, and fractures in community-dwelling older men. Aging Male. 2016;19(3):168-74.

\section{Publisher's Note}

Springer Nature remains neutral with regard to jurisdictional claims in published maps and institutional affiliations. 